\title{
Design of a Brain-Computer System to Measure Brain Activity during a Dolphin-Assisted Therapy using the TGAM1 EEG Sensor
}

\author{
Jaime Moreno $^{1}$, Oswaldo Morales ${ }^{1}$, Liliana Chanona ${ }^{1}$, Ricardo Tejeida ${ }^{2}$, \\ Pedro Flores ${ }^{3}$, Víctor Calderón ${ }^{4}$ \\ ${ }^{1}$ Instituto Politécnico Nacional, ESIME, Zacatenco, Mexico \\ ${ }^{2}$ Instituto Politécnico Nacional, EST, Mexico \\ ${ }^{3}$ Secretaría de Salud, Mexico \\ ${ }^{4}$ Delfiniti, S.A. de C.V. Ixtapa, Mexico \\ jemoreno@esimez.mx
}

\begin{abstract}
The recent proliferation of sensors technology applications in therapies to children disabilities to promote positive behavior among such children has produced optimistic results in developing a variety of skills and abilities in them. Dolphin-Assisted Therapy (DAT) has also become a topic of public and research interest for these disorders intervention and treatment. This work exposes the development of a system that controls brain-computer interaction when a patient with different abilities takes a DAT. The study was carried out at Definiti Ixtapa facilities and shows that brain activity increases by $376 \%$ during a DAT. A TAGM1 sensor was used to develop the system, which is connected to the Bluetooth 4.0 communication protocol, which is isolated from environmental conditions, which is brackish and humid. In this way, we explore the behavior of Obsessive Compulsive Disorder and neurotypic children using Fast Fourier Transform (FFT) from Electroencephalogram (EEG).
\end{abstract}

Keywords: artificial intelligence, EEG, FFT, TGAM1, dolphin-assisted therapy, BCI.

\section{Introduction}

Dolphins have had a therapeutic effect in the treatment of a number of human race conditions, both psychological and physical. Underwater communication sounds made by the dolphins play a part in this therapeutic effect. Dolphins appear to sense electrical fields from humans and attempt to communicate using the same frequencies. This has generated several aspects: (a) dolphin acoustic emissions, recorded in sea water, may bring about modifications in human brainwave activity, (b) dolphin interaction may give patients pain relief due to the increased release of hormones into the blood, (c) dolphin interaction may produce complex neurological, (d) stimulation which helps relaxation, helps reduce stress levels and thereby strengthens the immune system, and (e) dolphin's ultrasonic 
energy may cause significant cellular changes within the living tissue of the central nervous system [3].

Based on the above aspects, there has been developed an alternate treatment for people with diverse psychological and physical disabilities or disorders like Autism, Attention Deficit, Down Syndrome, Infantile Cerebral Palsy, and Obsessive Compulsive Disorder. This treatment is called Dolphin-Assisted Therapy (DAT). DAT is a growing area of interest to the general public, with reports of pain relief, extinction of depression and improved learning in children with different disabilities. [2]. DAT aims to improve functioning through complementing and reinforcing existing therapies, rather than replacing them [3].

In case of Obsessive Compulsive Disorder (OCD), this is characterized by intrusive, troubling thoughts (obsessions), and repetitive, ritualistic behaviors (compulsions) which are time consuming, significantly impair functioning and/or cause distress. When an obsession occurs, it almost always corresponds with a massive increase in anxiety and distress. Common obsessions include contamination fears, worries about harm to self or others, the need for symmetry, exactness and order, religious/moralistic concerns, forbidden thoughts (e.g., sexual or aggressive), or a need to seek reassurance or confess. Common compulsions include: cleaning/washing, checking, counting, repeating, straightening, routinized behaviors, confessing, praying, seeking reassurance, touching, tapping or rubbing, and avoidance. Unlike in adults, children need not view their symptoms as nonsensical to meet diagnostic criteria [6].

Younger children will not be able to recognize that their obsessions and compulsions are both unnecessary (e.g., you don't really need to wash your hands) and extreme (e.g., washing hands for 15-20 times is fine, but $5 \mathrm{~min}$ in scalding water is too much) in nature. In young children, compulsions often occur without the patient being able to report their obsessions, while adolescents are often able to report multiple obsessions and compulsions. Children and adolescents are also more likely to include family members in their rituals and can be highly demanding of adherence to rituals and rules, leading to disruptive and oppositional behavior and even episodes of rage [6].

A way the estimate the efficiency of DAT in children with different disabilities such as OCD is to observe changes in the children' brainwave activity from Electroencephalogram signals recorded before and during a session of DAT.

The electroencephalography (EEG) recorded from scalp give rise to the study of human cognitive activity through the measure of neurons' electrical impulses. At the beginning, the EEG data were often collected with the use of multiple electrodes and/or wired EEG headset to retain signal accuracy and spatial resolution, which greatly diminishes the mobility and actual application pragmatism. To facilitate a mobile driver safety program, [8] demonstrated the effectiveness of wireless single-channel (electrode) EEG headset NeuroSky's MindWave in distinguish user's eyes conditions - open or closed. 
Design of a Brain-Computer System to Measure Brain Activity during a Dolphin-Assisted ...

\section{Related Work}

For a System Artificial Intelligence is defined, it must include a model of the physical environment in which it is to operate [12]. In our case, we develop a model that measures the brain activity (physical feature) and the meaning of its behavioural changes. Thus, this proposal can be considered as an Artificial Intelligence model since it incorporates the knowledge of the domain of the application (in this case, in Neuroscience) to be able to measure the efficiency of the DAT from the study of biosignals.

For the study of these biosignals, it is necessary to use a Brain Computer Interface (BCI). In this way, BCI research programs have arisen due to more understanding of brain functions and powerful low cost computer equipment. Under BCI, the users can communicate with environments and the external devices through their brain activities. In particular, people suffering from disabilities like Obsessive Compulsive Disorder (OCD) have growing number of demands and needs of such system. The goal of these BCI systems are to translate the neuro-physiological signals for controlling the external devices. For acquiring the brain dynamics, electroencephalography (EEG) is relatively convenient, comfortable, and inexpensive [10].

To get the rid out of economical loss, but still taking in account the improvement in the reliability, accuracy, latency and false alarm rate, [4] have used the TAGM1 sensor as a probe to detect the brain wave pattern of the user and designed filter and threshold algorithm to detect driver's fatigues and drowsiness index with low cost and high reliability.

The aims of this work is trying to create a brain-computer system based on EEG. In this paper is developed a system to analyze and measure the both Obsessive Compulsive Disorder and neurotypic children brain activity during a DAT using the TGAM1 EEG signal sensor. Moreover, we measure, in a novel way, the Power Spectrum Density from EEG by means of BCI system, in order to estimate really changes in the children' brainwave activity recorded before, during and after a session of DAT. This paper is divided as follows: Section 3 introduces the system and architecture of BCI communication system; Section 4 describes the way we measure the brain activity before and during DAT; Section 5 shows the results and discussion and Section 6 is the conclusion.

\section{Theoretical Framework}

\subsection{Electroencephalography}

The brain is made up of hundreds of thousands of cells called neurons, which interact with each other in a bioelectrical phenomenon called synapses; By means of impulses that are transmitted around the membrane, information is sent that helps the brain to coordinate sensory, motor and cognitive functions. The activity of the present electric field can be manifested by a lapse of tens to hundreds of milliseconds, which is sufficient for equipment such as electroencephalographs, 
which use non-invasive electrodes on the cranial surface, to detect and record such activity.

Electroencephalography (EEG) studies, compared with other imaging techniques or behavioral observations, have advantages because of their excellent time resolution since several records can be taken in a second through multiple sensors. Other studies, such as MagnetoEncephaloGraphy (MEG), record the electric field activity generated by the neurons, have excellent resolution in time, but require large, stationary and expensive detectors, in addition to their maintenance and special training to operate such equipment. Functional Magnetic Resonance Imaging (fMRI) measures the change in blood flow associated with neuronal activity, since oxygenated blood, in comparison to the rest, causes magnetic distortions that are generated by the protons present in it, therefore fMRI has an excellent spatial resolution. Since human neuronal activity has a high degree of complexity in its nature, neuronal oscillations can be measured as a mixture of several underlying base frequencies, which reflect certain cognitive, attentional and affective states. These oscillations were defined in specific frequency ranges: delta, theta, alpha, beta and gamma.

- Delta (0.5-4 Hz): This type of wave has been examined during periods of deep sleep, locating its point of greater power in the right hemisphere of the brain. Since sleep is associated with the consolidation of memory, it plays a central role in learning functions. Its amplitude varies from $20-200 \mu V$.

- Theta $(4-8 \mathrm{~Hz})$ : Some studies report that the activity of this wave is related to cognitive activities such as: selective attention, assimilation of information, processing, learning and working memory. You can have a better record from the prefrontal, central, parietal and temporal zones. Its amplitude varies from $20-100 \mu V$.

- This oscillatory rhythmic activity is involved when there is a response to sensory stimuli, motor and memory functions. High levels of alpha can be noted when there is relaxation with closed eyes, the suppression of alpha implies a characteristic signature of mental states of interest, for example, when there is sustained attention to any type of stimulus at a particular time. Its amplitude varies from $20-60 \mu \mathrm{V}$ and the source of activation of these signals is in the occipital region of the brain

- Beta (12-25 Hz: This frequency is generated both in the occipital and frontal regions, it is associated with the states of active thinking, motor functions, visual and spatial coordination, anxiety and concentration. Its amplitude varies from $2-20 \mu V$.

- Gamma (more than $25 \mathrm{~Hz}$ ): They tend to have the highest frequency and the lowest amplitude. So far there is no strong association of what this type of waves reflect in the brain, there are some researchers who suggest being the reflection of the interconnection of several sensory responses to an object in a coherent way, therefore, represent a process of attention due to intense brain activities. On the other hand, others think that it is the effect of neural processes to achieve vision control, and, therefore, it would not represent a cognitive process after all. 
Design of a Brain-Computer System to Measure Brain Activity during a Dolphin-Assisted ...

There is discussion to date about a cerebral rhythm called mu $(\mu)$, its frequency band covers practically the same as alpha, however, it is only observed in the sensory-motor cortex.

\subsection{Electroencephalography Sensor: TGAM1}

MindWave Mobile by NeuroSky (Figure $1(\mathrm{~b})$ ) is Brain Computer Interface (BCI) in a headband that captures the EGG waves (Figure 1 (a)) and the eye blink of an user[7]. This device is an interesting for developers because they can program powerfull algorithms with an interface to connect to mobile devices easily and be able to use it in research applications [11].
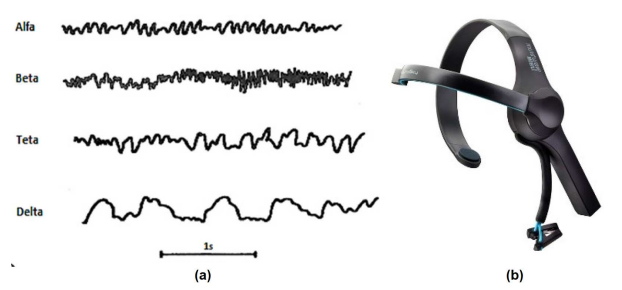

Fig. 1. (a) Types of EEG waves and (b) NeuroSky Mindwave Mobile.

From Figure 2, NeuroSky Mindwave Mobile can be divided in four parts:

1. Dry Electrode (Figure 4(b)),

2. Reference and Ground Electrodes (Figure 4(a)),

3. EEG biosensor TGAM1 (Figure 2(c)), and

4. Communication Module (Figure 2(c)).

NeuroSky's EEG biosensor digitizes and amplifies raw analog brain signals to deliver concise inputs the most important features of this biosensor are the following:

- Direct connect to dry electrode since it does not need a special solution or gel,

- One EEG channel, Reference, and Ground,

- Extremely low-level signal detection,

- Advanced filter with high noise immunity, and

- RAW EEG at 512Hz.

The code that may appear in the ThinkGear packets are listed in the Table 1.

TGAM1 has configuration pads that can be used to change two default settings that are applied at chip power up. The configuration pads are located on 

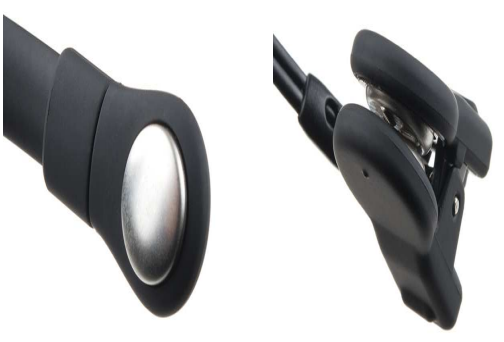

(a) Electrode.
Dry

(b) and Electrodes.

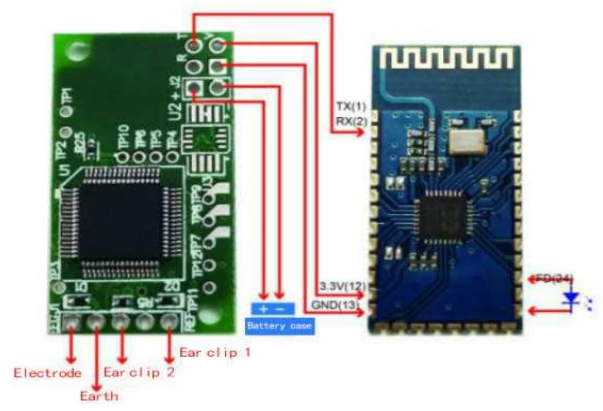

(c) EEG biosensor TGAM1 with CommunicaGround tion Module.

Fig. 2. NeuroSky Mindwave Mobile system main parts.

Table 1. ThinkGear CODE.

\begin{tabular}{cclc}
\hline \multicolumn{2}{c}{ Code Length } & \multicolumn{1}{c}{ Value } & Default Setting \\
\hline 0x02 & N/A & Poor Quality (0-200) & On \\
0x04 & N/A & eSense Attention (0-100) & On \\
0x05 & N/A & eSense Meditation (0-100) & On \\
0x80 & 2 & 10-bit Raw EEG & Off \\
0x83 & 24 & EEG Powers (integer) & On \\
\hline
\end{tabular}

the backside of the TGAM1, as indicated by the red square in Figure 3(a). From Table 2, the BR0 and BR1 pads configure the output baud rate and data content, after the TGAM1 powers up. The M pad configure the notch filter frequency. Normal Output mode includes the following output: poor quality value, EEG value, Attention value and Meditation value.

A magnified picture of the B1 and B0 pads are shown in Figure 3(b). The first row of pads are GND and third row of pads are VCC. The TGAM1 output baud rate and data content after power up behavior depends on the pad setting as described in table above. For example, the stuff option in the module in Figure 3(a) has both BR1 and BR0 tie to GND pads for a 9600 baud with Normal Output Mode.

The baud rate can also be configured after the module is powered up by sending commands through the UART interface. The commands are listed in the Table 3 . When the module is reset, the baud rate setting will revert back to the default set by BR0 and BR1.

TGAM1's notch filter frequency can be configured with the M configuration 


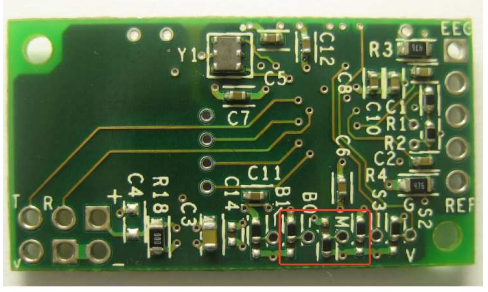

(a) Main Pads

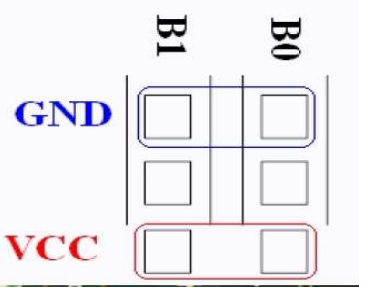

(b) B0 and B1.

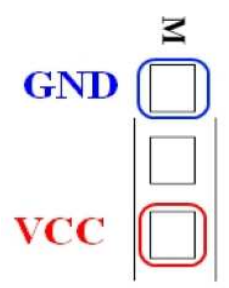

(c) M.

Fig. 3. Configuration pads in TGAM1 chip.

Table 2. Configuration pads.

\begin{tabular}{l}
\hline BR1 BR0 \\
\hline GND GND 9600 Baud with Normal Output Mode \\
GND VCC 1200 Baud with Normal Output Mode \\
VCC GND 57.6k Baud with Normal + Raw Output Mode \\
VCC VCC N/A
\end{tabular}

Table 3. Baud rate configuration.

\begin{tabular}{cc}
\hline Command & Function \\
\hline 0x00 & 9600 Baud with Normal Output Mode \\
0x01 & 1200 Baud with Normal Output Mode \\
0x02 & 57.6k Baud with Normal + Raw Output Mode
\end{tabular}

pads. It is used to select either $50 \mathrm{~Hz}$ or $60 \mathrm{~Hz}$ to reduce the $\mathrm{AC}$ noise specific to a targeted market. As indicated in Figure 3(c), the top pad is GND and bottom pad is VCC. Tie the M pad to VCC pad to select $60 \mathrm{~Hz}$, and to GND pad to select $50 \mathrm{~Hz}$ notch filtering frequency.

Unlike the BR0, BR1 configuration, there is no equivalent software configuration for the $\mathrm{M}$ configuration. The most common stuff option for these configuration pads are illustrated in Figure 3(a), configuring the TGAM1 for 9600 Baud, normal output and $60 \mathrm{~Hz}$ notch filtering frequency.

\subsection{Filtering Biosignals using Fast Fourier Transform}

The Fast Fourier Transform (FFT) algorithm is based on the method known as successive bending. It is defined by Equation 1 as:

$$
F_{u}=\frac{1}{2 \cdot M}\left[\sum_{x=0}^{N-1}\left(a_{x} \cdot w(u, x)\right)\right]
$$


where $w(u, x)$ can be defined by Equation 2 as following:

$$
w(u, x)=e^{\left(-j \cdot 2 \cdot \frac{u \cdot \pi \cdot x}{2}\right)} .
$$

The result of the transformation is represented in a vector called $F$ of subscript $u$. The value $N$ is the amount of element of the sample, $M$ is half of $N$. The values of the sample are loaded in the vector called $a_{x}$ and $x$ represents the subscript. Equations 3 and 4 are obtained from Equation 1:

$$
\begin{gathered}
\text { Feven }_{u}=\frac{1}{M} \cdot\left[\sum_{x=0}^{M-1}\left(a_{2 \cdot x} \cdot w_{1}(u, x)\right)\right], \\
\text { Fodd }_{u}=\frac{1}{M} \cdot\left[\sum_{x=0}^{M-1}\left(a_{2 \cdot(x+1)} \cdot w_{1}(u, x)\right)\right] .
\end{gathered}
$$

All the even subscripts of $x$ belonging to $a_{x}$ are grouped in the vector Feven and the odd ones in the vector Fodd. In this case $w_{1}(u, x)$ is defined by Equation 5 as:

$$
w_{1}(u, x)=e^{\left(-j \cdot 2 \cdot \frac{u \cdot u \cdot x}{\frac{N}{2}}\right)} .
$$

Frequently, the relevant information of a signal has a characteristic wave that is known in a general way, for this it is possible to obtain time series containing this information, later study measures of relations between signals in both the time domain and the frequency (correlation or crossed spectrum) and more complex transformations for the extraction of characteristics.

In the case of EEG signal analysis, considering the time plotted on the $X$ axis and the voltage on the $Y$ axis; The Fast Fourier Transform transforms the signal from the time domain to the frequency. Basically what is done during this transformation is to examine the raw data of the signal how much can be approximated to sine waves consisting of pure frequencies, that is, free of redundancy; the more they adjust, the greater their correlation. Since the frequencies of the human brain associated with affective and cognitive activities are in the range of $0.5-60 \mathrm{~Hz}$, an analysis by the FFT gives a lot of relevant information, for example, if a person is in a general state of concentration (theta band) or the response of their neuronal activity describes a dream state (delta band).

To implement the FFT in the microcontroller it is necessary to implement Feven and Fodd. We used MatLab 2018b to implement both the programming of the embedded devices and the analysis of these signals. 
Design of a Brain-Computer System to Measure Brain Activity during a Dolphin-Assisted ...

\section{Measuring Brain Activity during a Dolphin-assisted Therapy: DAT-TGAM1 System}

\subsection{Designing of the Waterproof Case}

The original BCI adaptation consists of keeping the headband but introducing the TGAM1 sensor in a waterproof case, Figure 4 shows the Modeling and Printing of this case.

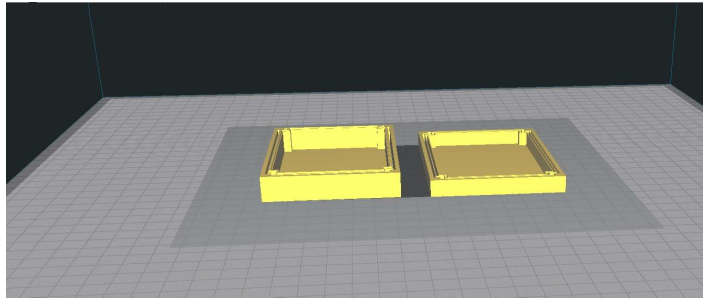

(a) 3D Modeling and Designing.

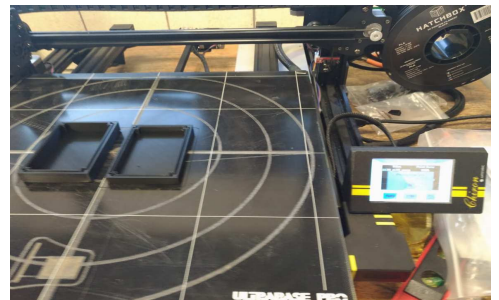

(b) 3D Printing.

Fig. 4. Designing of the waterproof case where TGAM1 Microcontroller is located.

An important part of the choice of material for 3D printing are the parameters of the water quality of confinements in closed facilities must meet the following characteristics:

- Salinity: 18 to 36 parts per thousand.

- Hydrogen Potential (pH): between 6 and 8 units.

- Temperature: from 5 to $27^{\circ} \mathrm{C}$.

- Pressure: 1 Atmosphere (ATM).

It is important to mention, even this case supports at least one atmosphere of pressure, that is to say, that it does not leak at 10 meters of depth, it was designed for floating.

\subsection{Processing EEG Signals}

For this work, we explore the behavior of two children (control and intervention patients) using the quantitative mathematical tool such as Fast Fourier Transform (FFT) from ElectroEncephaloGram (EEG) signals[1]. The EEG RAW data are time series that showed the cerebral brain activity, voltage versus time, at rest or before DAT (Figure 5(a)), during a DAT (Figure 5(b)), and after DAT (Figure $5(\mathrm{c})[9])$. As recorded by the first frontopolar electrode $\left(F_{P 1}\right)$ by means of a EEG biosensor TGAM1 Module.

For analyzing EEG data we decomposed the signal into functionally distinct frequency bands: $\delta(0.5-4 \mathrm{~Hz}), \theta(4-8 \mathrm{~Hz}), \alpha(8-12 \mathrm{~Hz}), \beta(12-30 \mathrm{~Hz}), \gamma$ 


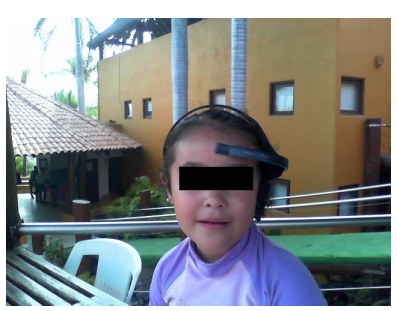

(a) Before DAT

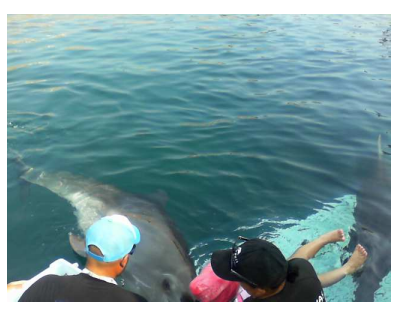

(b) During DAT

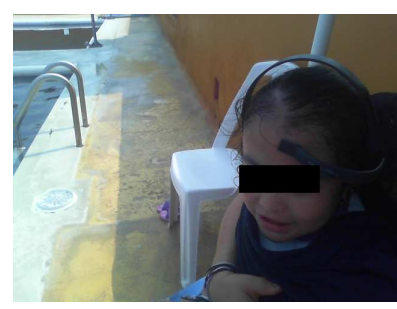

(c) After DAT.

Fig. 5. Method of obtaining EEG RAW samples.

$(30-60 \mathrm{~Hz})$, and All Bands $(0.5-60 \mathrm{~Hz})$ through FFT, in order to obtain an estimate of the Power Spectral Density (PSD), expressed in $\mu$ Volts $/ \mathrm{Hz}[5]$.

\section{Experimental Results}

\subsection{Initial Conditions}

The main goal of this proposal is to test our BCI when a patient who has a problem in neurodevelopment, we have chosen a patient with Obsessive Compulsive Disorder (OCD), who is taking a Dolphin Assisted Therapy (DAT). The whole system is subdivided into three fundamental parts:

1. Female dolphin of the bottle nose species

2. Patient with OCD.

3. DAT-TGAM1 system.

All three subsystems interact in order to determine if DAT is effective for OCD patients

\subsection{Results}

Figure 6 shows the results of our experiments for measuring the efficiency of DAT for RAW EEG Brain activity (first row) of the control and intervention child patients, first and second columns respectively, using FFT - Power Spectrum Density (second and third rows) Before DAT, During DAT and After DAT. In this image we can realize that there are meaningful changes in behavior experienced by these two-treated children. Figures 6(a) and 6(b) show that there is a higher brain activity during DAT than Before or After, namely, FFT analysis points out an average great increment of $376 \%$ in the average power spectral density of data in both patients during the DAT respect to before it, which yields the increment on every spectral band, Figures 6(c) and 6(d). While Figures $6(\mathrm{e})$ and $6(\mathrm{f})$ show the entire power spectral density from 0 to $256 \mathrm{~Hz}$ (half of $\delta_{t}$ ) and it can be noticed that the overall power is higher in all cases when a DAT is developed. 


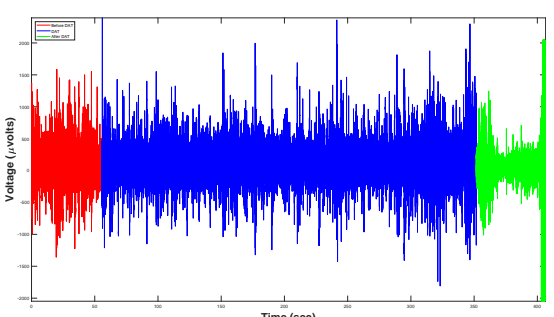

(a)

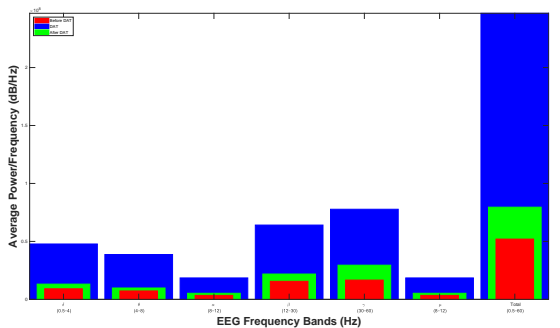

(c)

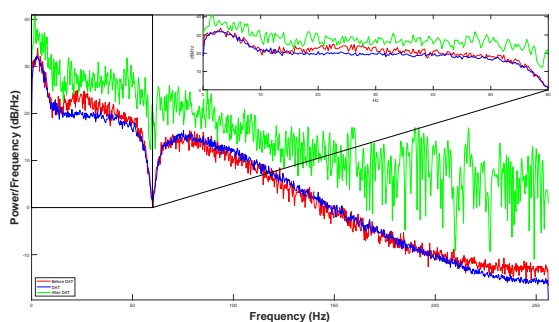

(e)

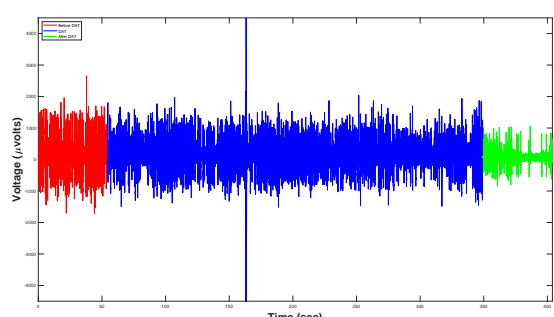

(b)

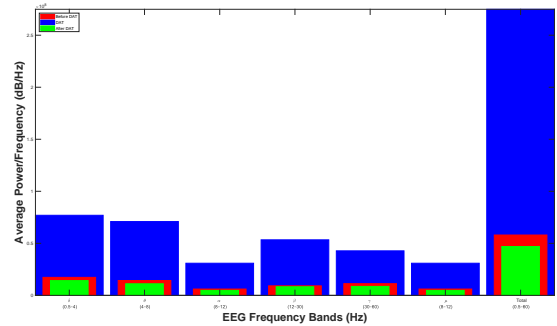

(d)

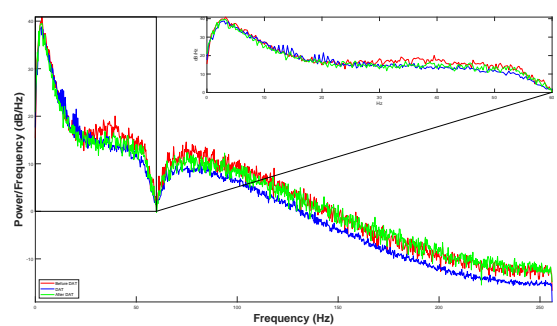

(f)

Fig. 6. Results of efficiency of DAT for RAW EEG Brain activity (first row) of the control and intervention child patients, first and second columns respectively, using FFT - Power Spectrum Density (second and third rows) Before DAT, During DAT and After DAT.

\subsection{Discussion}

Table 4 is the summary of the experimental results. When we compare Average Power Spectrum Density (PSD) During DAT regarding before it, we can realize that the brain of the patients increment its activity in approximately $376 \%$ in both cases. After the DAT, the brain activity of the control patient was reduced in $67.92 \%$, while the intervention patient reduces its activity in $83.02 \%$, namely, the patient with OCD gets an apparent relaxation since its PSD decreases $20 \%$ regarding its initial rest state meanwhile the control patient increases its PSD $53 \%$ regarding its initial rest state. 
Table 4. Summary of the experimental results.

\begin{tabular}{lccc}
\cline { 2 - 4 } & \multicolumn{3}{c}{ Power Spectrum Density } \\
\cline { 2 - 4 } & BEFORE & DAT & AFTER \\
\hline Control Patient & 15345000 & 73158000 & 23470000 \\
\hline Intervention Patient & 17433000 & 82959000 & 14088000 \\
\hline
\end{tabular}

\section{Conclusions}

Brain Computer Interface (BCI) can have variety of application areas such as Neuroscience research, where it can help Obsessive Compulsive Disorder and neurotypic children where its sources mainly include EEG signals obtained from brain using head sensors such as the TAGM1, used to develop the proposed system in this paper.

Based on the outcomes from the PSD analysis, we obtain the results of the Table 4 which show that with this mathematical method, there is a huge increment of brain activity during DAT regarding the other two states, before and after therapy.

Our results show the efficiency of DAT is around of the $376 \%$ of increment of neuronal PSD. The novelty of the PSD analyses in a TGAM1 EEG sensor is the ability to identify and give a mathematical meaning of the beneficial changes in behavior of the neuronal system of children with or without Obsessive Compulsive Disorder when their treatment is assisted by a bottle-nose dolphin.

Finally, our findings point out an increment of neural activity (more PSD) during DAT, displaying collective behaviors, i.e., positive increments of neuronal activity could be followed by much more neural activity. Thus, the brain could react to DAT gradually over a period of time, which indicates the existence of external features that modify the brain behaviour of children with diverse psychological and physical disabilities or disorders.

Acknowledgment. The research described in this work was carried out at Delfiniti México, Ixtapa in collaboration with the Instituto Politécnico Nacional and the Secretaría de Salud of Mexico by means of projects No. 20190046 and 20195208. Also, Hugo Quintana, Ixchel Lina, Teresa Ivonne Contreras, Ana Gabriela Ramírez, Alfredo Durand, and Pedro Arrechea are thanked for the methodological and technical support.

\section{References}

1. Abtahi, F., Ro, T., Li, W., Zhu, Z.: Emotion analysis using audio/video, emg and eeg: A dataset and comparison study. In: 2018 IEEE Winter Conference on Applications of Computer Vision (WACV). pp. 10-19 (March 2018) 
2. Birch, S.: Dolphin sonar pulse intervals and human resonance characteristics. In: Proceedings of the 2nd International Conference on Bioelectromagnetism (Cat. No.98TH8269). pp. 141-142 (Feb 1998)

3. Chengwei, L., Xiaoming, H., Limei, Z.: The study on brain paralysis ultrasonic therapy instrument simulating dolphin. In: 2005 IEEE Engineering in Medicine and Biology 27th Annual Conference. pp. 6056-6059 (Jan 2005)

4. Joshi, D.H., Jaliya, U.K., Thakore, D.G.: A.r.g.o.s: Alertness rating gamma brainwave observation system. In: 2016 International Conference on Data Mining and Advanced Computing (SAPIENCE). pp. 374-377 (March 2016)

5. Kantelhardt, J.W., Tismer, S., Gans, F., Schumann, A.Y., Penzel, T.: Scaling behavior of eeg amplitude and frequency time series across sleep stages. EPL (Europhysics Letters) 112(1), 18001 (October 2015)

6. Lack, C.: Obsessive-compulsive disorder: Evidence-based treatments and future directions for research. World journal of psychiatry 2, 86-90 (12 2012)

7. Li, K.G., Shapiai, M.I., Adam, A., Ibrahim, Z.: Feature scaling for eeg human concentration using particle swarm optimization. In: 2016 8th International Conference on Information Technology and Electrical Engineering (ICITEE). pp. 1-6 (Oct 2016)

8. Lim, C.K.A., Chia, W.C., Chin, S.W.: A mobile driver safety system: Analysis of single-channel eeg on drowsiness detection. In: 2014 International Conference on Computational Science and Technology (ICCST). pp. 1-5 (Aug 2014)

9. Senevirathna, B., Abshire, P.: Spatio-temporal compressed sensing for real-time wireless eeg monitoring. In: 2018 IEEE International Symposium on Circuits and Systems (ISCAS). pp. 1-5 (May 2018)

10. Singh, A.K., Wang, Y.K., King, J.T., Lin, C.T., Ko, L.W.: A simple communication system based on brain computer interface. In: 2015 Conference on Technologies and Applications of Artificial Intelligence (TAAI). pp. 363-366 (Nov 2015)

11. Sosa-Jimenez, C.O., Mesa, H.G.A., Rebolledo-Mendez, G., de Freitas, S.: Classification of cognitive states of attention and relaxation using supervised learning algorithms. In: 2011 IEEE International Games Innovation Conference (IGIC). pp. 31-34 (Nov 2011)

12. Wichmann, T.: Detail zooming in artificial intelligence world modeling. In: SoutheastCon 2015. pp. 1-5 (April 2015) 